\title{
Pace of life and perceived stress in international students
}

\author{
Sonia Lippke (1), ${ }^{1}$ Torven M. Schalk, ${ }^{1,3}$ Ulrich Kühnen, ${ }^{1}$ and Borui Shang ${ }^{2}$ \\ ${ }^{1}$ Department of Psychology and Methods, Jacobs University Bremen, Bremen, Germany, ${ }^{2}$ Department \\ of Social Sciences, Hebei Sport University, Shijiazhuang, China, ${ }^{3}$ School of Psychology, Victoria \\ University of Wellington, Wellington, New Zealand
}

\begin{abstract}
An accelerated pace of life greatly impacts individuals' health and lifestyles. However, this imposition has not been systematically researched within a culturally diverse sample. Thus, this study aimed to explore the subjective experience of the pace of life and its correlates in a culturally diverse sample within a German university context. This was done to test whether students $(N=156)$ with a migration background from other countries $(n=105)$ differ from students without migration background $(n=51)$. The pace of life, life satisfaction, stress, work-life balance, and health were measured on an individual level along with sociodemographic variables through online questionnaires. The pace of life was found to be invariant across students from different cultural backgrounds and unrelated to the length of stay at the current university. Interrelations were found between pace of life and work-life balance, $r=.21, p<.05$. While this study revealed perceived stress to be generally prevalent among students, the relationship between a slower pace of life and increased stress levels, $\beta=-.17, p<.05$, disappears when controlling for health, $\beta=-.26, p<.01$, work-life balance, $\beta=-.28, p<.01$, and life satisfaction, $\beta=-.25, p<.01$, as well as sociodemographic variables (only gender and medium length of stay were significant.) Furthermore, a mediation effect, $b=-1.89,95 \%$ CI $[-3.598,-0.463]$, revealed that students cope with a faster pace of life by effective time management, which also leads to better work-life balance and in turn reduces the experienced level of stress. Future research should examine psychological mechanisms more extensively in longitudinal research and apply interventional designs to help students prevent and manage stress in the era of a fast-paced life.
\end{abstract}

Keywords: diverse cultural backgrounds; pace of life; stress; well-being; work-life balance

Correspondence Prof. Dr. Sonia Lippke, Department of Psychology \& Methods/Focus Area Diversity, Jacobs University Bremen gGmbH, Campus Ring 1, 28759 Bremen, Germany. Email: s.lippke@jacobs-university.de

Received 23 May 2020. Accepted 7 December 2020.

For many individuals, life is becoming increasingly faster, and they are having less and less time available (Burnett, DiTunnariello, \& DeGreeff, 2020; Levine \& Norenzayan, 1999). The pace of life is faster in countries with colder climates, and in individualist (vs. collectivist) and economically productive cultures (Levine \& Norenzayan, 1999). Gleick (1999) aptly illustrated these phenomena in his bestseller Faster: The Acceleration of Just About Everything. In modern societies, citizens seem to experience an increase in time pressure and progressively feel more deprived of time (Keinan, Bellezza, \& Paharia, 2019).

The accelerating pace of life is noticeable in our everyday life and specifically at work, where new job demands are placed on employees, especially by introducing new information and communication technologies (Nagy, Eschrich, \& Finn, 2020). The same holds true for university students (e.g., Goldbach, Hoffmann, Hoppe, Pitz, \& Thommes, 2020; Güzel \& Glazer, 2019). Possible hypotheses about the consequences of an accelerated pace of life might be deduced from previous studies that have found a link to different health indicators such as cancer, coronary heart diseases (CHDs), and well-being (e.g., Levine \& Norenzayan, 1999; Melnikov, Krzhizhanovskaya, Lees, \& Sloot, 2020; Royauté, Berdal, Garrison, \& Dochtermann, 2018; Shi et al., 2020) as well as health-related behavior (i.e., risky driving, Goldbach et al., 2020). 


\section{Definition and operationalization regarding the pace of life}

Werner, Altman, and Oxley (1985), provided a wellacknowledged definition of the pace of life as the "relative rapidity or density of experiences, meanings, perceptions and activities" (p. 14). The pace of life has frequently been operationalized on the level of entire collectives such as cities or countries through walking speed, work speed of postal clerks, and accuracy of public clocks (e.g., Bornstein \& Bornstein, 1976; Levine \& Bartlett, 1984). A fast pace of life has been shown to be associated with poor health-again measured on the level of whole collectives (e.g., prevalence of CHDs; Levine \& Norenzayan, 1999). On a sociological level, Levine, Lynch, Miyake, and Lucia (1989) provided support for the contention that the pace of life is one component of Type A behavioral patterns. These authors also pointed to the need to develop measurements on the individual level to improve the study of these relations. One of these individual-level measures is the concept of time urgency (Goldbach et al., 2020; Mathot \& Frankenhuis, 2018), yet further evidence is required.

\section{Pace of life related health indicators}

Previous studies have shown that the pace of life varies cross-culturally. Levine and Bartlett (1984) studied the pace of life in cities across six countries and found positive correlations of the pace of life with deaths from CHDs in Western countries (United States, England, Italy). However, in Japan, one of the most fast-paced countries widely acknowledged, individuals there had the lowest number of deaths caused by stress-related diseases such as CHD. In addition, the behavioral pattern connected to the pace of life has also been investigated. For instance, a review study revealed that Type A behavior and its related diseases are positively related to a fast pace of life (Rosenman, 1990). Other authors have detected a positive correlation between pace of life and stress (Melnikov et al., 2020) or CHD death rates across 36 cities in the United States (Levine et al., 1989). Levine et al. (1989) concluded that their results support the concept of "Type A cities;" that is, "metropolitan areas with behavioral norms which create a predisposition to coronary heart disease" (p. 518).
Levine and Norenzayan (1999) extended their previous research by enlarging the samples across cities in 31 countries, examining a broad range of factors related to the pace of life. The results showed that the pace of life is slower in collectivistic cultures (e.g., Japan, Korea, China) than individualistic ones (e.g., North American and European countries). In addition, the pace of life positively correlated with deaths from CHD. Recently, it was found that especially those college students in China who have difficulties in adapting to the pace of their campus life are also more likely to suffer from back pain (Mei et al., 2019). In sum, a fast pace of life seems to be related to several health risks (Royauté et al., 2018).

\section{Pace of life and related psychocultural variables}

The pace of life has also been found to be related to various forms of psychological and physical well-being. Life-satisfaction is an important factor that has received the attention of researchers. Although a fast pace of life seems to be connected to negative health outcomes, it has also been linked with increased subjective well-being and life satisfaction (Levine \& Norenzayan, 1999). However, other research has shown no such relationship (van de Vliert \& Janssen, 2002). Furthermore, a fast pace of life has been connected to higher stress levels (Garhammer, 2002; Melnikov et al., 2020), and higher stress levels, especially among students, were related to decreased life satisfaction (Cotton, Dollard, \& de Jonge, 2002).

These mixed results on the relationship of the pace of life and life satisfaction underline the importance of research in this area. In addition, it has been indicated that a fast pace of life is related to an inability to maintain a healthy work-life balance (Levine \& Bartlett, 1984). It is assumed that individuals with a fast pace of life always feel the urgency to quickly complete the assigned tasks, thus making them unable to separate their work from their personal life. However, given the correlational nature of the evidence, the causal direction remains unclear. It may well be that individuals with more efficacious behavior feel less stressed and therefore have more time to better manage their tasks. In line with this contention, effective time management has been shown to be related to undergraduate engineering students' performances measured in grades (Adams \& Blair, 2019). 
Furthermore, acculturation has also been previously linked with the pace of life. Acculturation has been defined as "the phenomenon occurred when groups of individuals having different cultures come into continuous first-hand contact, with subsequent changes in the original cultural patterns of either or both groups" (Redfield, Linton, \& Herskovits, 1936, p. 149). Over time, migrants adapt psychologically to fit in a new environment, and the pace of life is one of the domains important for sociocultural adaptation and, hence, the part of scales that are used to measure such adaptation (Güzel \& Glazer, 2019; Ward \& Kennedy, 1999; Wilson, Ward, Fetvadjiev, \& Bethel, 2017). Thus, adaptation to the new culture should be considered, too, and is included in the current study as the length of stay at the current university in accordance with prior research that has shown that the length of stay is positively related with acculturation (e.g., Wang \& Mallinckrodt, 2006; Wilton \& Constantine, 2003).

\section{Pace of life related research in students}

A fast pace of life may lead to increased stress, and increased stress can be related to decreased satisfaction (e.g., Levine \& Norenzayan, 1999). Previous studies investigating stress among students have mostly focused on the prevalence of high stress levels, their consequences, and on students' coping mechanisms. A recent study (Xing, Popp, \& Price, 2020) pointed out that adaption to a new environment is crucial in slowing down the pace of life. In other words, Chinese students studying in the United States who adapted socially and psychologically to the pace of life were much better off than those who did not adapt well (Xing et al., 2020). In addition, stress was found to be a risk factor for poor mental health, and more than $80 \%$ of students in a study by the American College Health Association (2018) reported feeling overwhelmed by all they had to do, with more than 1 in 10 having seriously considered suicide.

In a study on the sources of stress, $78 \%$ of university students were found to be under moderate stress and another $10 \%$ under serious stress, with the main sources of stress being academic stressors (e.g., lack of time for study) and life stressors (e.g., lack of time for family, friends, and own interests). These findings highlight the need for further research on the causes of high stress at universities and to facilitate the development of effective preventive measures.
However, many studies did not investigate the origins and intercorrelates of the stressors. It is therefore imperative to systematically examine the interrelations among the pace of life, stress, health, and well-being in an international university context with students having differing adaptions in terms of their length of stay at the current university.

\section{Research aims and questions}

The present study was exploratory in nature and measured the pace of life at an individual level in a culturally diverse sample of university students. The research aims of this study were (a) to assess the descriptive features of a pace of life in the given sample and whether there are differences across countries of origin; (b) to explore interrelations among pace of life with the variables of stress, worklife balance, health, and life satisfaction; and (c) to examine which variables predict stress with special focus on the pace of life, health, work-life balance, and life satisfaction after controlling for sociodemographic variables (age, gender, country of origin, and study background) and adaption in terms of the length of stay at the current university.

The study focused on university students for several reasons. First, previous research has shown that a large share of university students generally suffer from high stress levels with potentially detrimental consequences for health and well-being (Pascoe, Hetrick, \& Parker, 2020). Second, an international university context is characterized by high cross-cultural diversity, allowing one to investigate potential cultural differences in the variables under consideration. Specifically, we were interested in whether international students differ from domestic ones in the pace of life. In line with the aforementioned research aims, the research questions are:

Research Question 1 (RQ1): Does the pace of life significantly vary across different countries of origin (i.e., students with migration background from foreign countries and local students without migration background)?

Research Question 2 (RQ2): Does the pace of life on an individual level significantly correlate with the four following variables: (a) life satisfaction, (b) stress level, (c) work-life balance, and (d) health?

Research Question 3 (RQ3): Which of the variables (countries of origin, life satisfaction; work-life balance, and health) explain the most variance in stress after testing 
the impact of the pace of life among students and controlling for age, gender, adaption, and study subject?

\section{Method}

Data on the pace of life, stress, work-life balance, health, and well-being were collected on an individual level from a culturally diverse sample in a university in 2017. Online self-report questionnaires were collected via the online survey software UniPark (Online Questionnaire Software Quest Back, Unipark). On the first page of the survey, all participants were informed about the confidentiality, anonymity, and voluntary nature of their responses, and were given the opportunity to obtain the study results by providing contact information on the page. Those who provided their informed consent could continue to the questionnaire pages. The study received ethical approval by the ethics committee of the German Psychological Society (Deutsche Gesellschaft für Psychologie, EK-A-SL022013). The link for the survey was sent to potential study participants.

\section{Participants}

By convenience sampling, participants were recruited via repeated email messages through the university mailing lists as well as Facebook posts in relevant groups of students to recruit as many participants as possible. A total of 205 students were recruited as participants, and 156 of them completed the online survey (completion ratio $=76.1 \%$ ). Mean age of the sample was 20.45 years $(S D=2.06)$, and $54.5 \%$ of the participants were female (44.9\% male; $0.6 \%$ other). Respondents identified themselves in total with 45 different countries of origin, with Germany being the most frequent one $(n=51)$. Participants studied one of the three major social sciences in almost equal proportions $(n=55)$, natural sciences $(n=48)$, and informatics, mathematics, and logistics $(n=53)$. A similar distribution emerged concerning the length of stay at the current university, with 55 first-year students, 49 secondyear students, and 52 third-year students.

\section{Questionnaire construction}

The questionnaire used in the present study was constructed from a review of the relevant literature as well as existing scales. In total, it included 34 items in six sections: the pace of life (10 items), life satisfaction ( 2 items), stress (10 items), work-life balance (5 items), health ( 2 items), and sociodemographics (5 items). The measures of each section are specified next.

\section{Pace of life}

The pace of life was measured by items representing a range of behaviors that are related to the pace of life, with answers ranging from 1 (e.g., never) to 5 (e.g., all day). This was a self-developed Likert scale consisting of 10 items. Four items were concerning information and communication technology related behaviors (adapted from Thomeé, Härenstam, \& Hagberg, 2011), one for each behavior. Previous studies have indicated relationships between the pace of life and six further behaviors, each measured by a single item: regarding dieting (Garhammer, 2002; Levine, 1997; Rosa, 2003), sleeping (e.g., Rosa, 2003), multitasking (Garhammer, 2002; Sullivan \& Gershuny, 2001), punctuality (Levine, 1997; Levine \& Bartlett, 1984), leisure activities (e.g., Sullivan \& Gershuny, 2001), and irritation frequency (Levine, 1997).

The main focus for the analysis lies in the individual items and their relationships with the well-being variables. In addition, the scores of the pace of life items were aggregated to investigate possible relationships that extend across individual behavior. This was subsequently computed as the arithmetic mean from 1 (lowest pace of life) to 5 (fastest pace of life). Therefore, the analysis includes the pace of life aggregate and the individual items from which it was constructed. This was done to identify the relationships of each of the items with the well-being variables. Additionally, two more items were added in the pace of life section that asked participants for their assessment of their own pace of life in comparison to the pace of life at university as well as to the pace of life at home. These two items were not included in the pace of life aggregate but were used for individual analysis.

\section{Life satisfaction}

Life satisfaction was measured by the Satisfaction With Life Scale (SWLS; Pavot \& Diener, 2008) on a five-item scale (e.g., "How satisfied are you with your health?" and "How satisfied are you with your sleep?"). The scores were created by summing up scores of the respective items for each participant, resulting in a possible score range of 5 (lowest life satisfaction) to 25 (highest life satisfaction). SWLS has been confirmed to be a reliable measure for life satisfaction, with Cronbach's $\alpha$ of .84 indicating a good internal consistency. 


\section{Stress}

Stress was measured by the Perceived Stress Scale (PSS; Cohen, Kamarck, \& Mermelstein, 1983). The scale used in this study is the 10-item version of the PSS (Cohen \& Williamson, 1988; "In the last month, how often have you been upset because of something that happened unexpectedly?" "In the last month, how often have you felt that you were unable to control the important things in your life?" "In the last month, how often have you felt nervous and "stressed"?" "In the last month, how often have you felt confident about your ability to handle your personal problems?" "In the last month, how often have you felt that things were going your way?" "In the last month, how often have you found that you could not cope with all the things that you had to do?" "In the last month, how often have you been able to control irritations in your life?" "In the last month, how often have you felt that you were on top of things?" "In the last month, how often have you been angered because of things that were outside of your control?" "In the last month, how often have you felt difficulties were piling up so high that you could not overcome them?").

The PSS measures if situations are appraised as stressful by the respondent rather than assessing psychiatric symptoms of stress, hence giving a subjective insight from the individual perspective. It has been validated in the population of college students (Roberti, Harrington, \& Storch, 2006). PSS scores could range between 0 (lowest stress levels) and 40 (highest stress levels). In a normative data from young individuals aged 18 to 34 years, the mean was $15.60(S D=6.67)$ (Nordin \& Nordin, 2013). Cronbach's $\alpha$ for the PSS was .87 , indicating a good internal consistency.

\section{Work-life balance}

Work-life balance was measured by the translated version of the Trierer Scale to Measure Work-Life Balance (TKSWLB; Syrek, Bauer-Emmel, Antoni, \& Klusemann, 2011) consisting of five items ("I am satisfied with my balance between work and private life.." "I find it difficult to balance work and private life." "I can manage challenges of my private life and challenges of my professional life equally well." "It's possible for me to achieve a good balance in life between stressful and relaxing activities." "I am satisfied with how my priorities in relation to the profession and private life are divided."). The TKS-WLB scores were calculated, and possible scores ranged between 1 (worst work-life balance) and 5 (best work-life balance). The scale showed a Cronbach's $\alpha$ of .81, indicating good reliability.

\section{Health}

A subjective measure was used for assessing the general health, satisfaction with health, and satisfaction with the sleep of the participant. Three items were selected from the SF-36 Health Survey (Ware \& Sherbourne, 1992) and the WHOQOL-BREF (Whoqol Group, 1998)_- “How satisfied are you with your health?" "How satisfied are you with your sleep?" "How healthy do you feel?"- - and were rated on a scale of 1 (unhealthy) to 5 (healthy). The average health rating was calculated by averaging the answers to the respective items, resulting in possible scores of 1 (lowest health level) to 5 (highest health level). With a Cronbach's $\alpha$ of .74, the scale reached acceptable reliability.

\section{Sociodemographics}

Age, gender, major, country of origin, and adaption/length of stay at the current university were asked for each respondent (five items total). Particularly for the country of origin item, there was no scale measuring a collectivist or an individualist orientation to not overload the questionnaire and to retain participant's interest. Instead, participants were asked for the country to which they feel most closely connected. This was done because many participants are so-called "third-culture kids;" thus, the country of origin might not be the most accurate indicator of participants' cultural orientation. According to the mentioned country, each respondent was assigned the respective individualism-collectivism score based on research by Hofstede (2001) and Hofstede, Hofstede, and Minkov (2010). To assess at least some aspects of adaption, we simply asked how many years the participant had stayed at the current university as a proxy.

\section{Analysis}

All analyses were run in IBM SPSS Version 23. Descriptive statistics were used to assess the pace of life, perceived stress, work-life balance, life satisfaction, and health. Correlational analyses were employed to explore the interrelations among the pace of life, the well-being scales, the country of origin, and age. For the interpretation of the findings, we have followed recommendations by 
Cohen (1992): Correlations of $r=.10$ are considered small, $r=.30$ as medium, and $r=.50$ as large.

Hierarchical regressions were used to explore the relationship among perceived stress (as the dependent variable), sociodemographic variables (age, gender, country of origin, adaption, study major; Block 1 in the regression model), the pace of life (Block 2), and health-related variables (health, work-life balance, life satisfaction; Block 3). A subsequent mediation analysis was run employing the PROCESS macro in SPSS (PROCESS Model 4; Hayes, 2018).

\section{Results}

\section{Descriptive statistics and correlational analyses among health-related variables}

Table 1 shows the descriptive statistics $(M$ and $S D)$ of all scales used in this study. Correlational analyses of the wellbeing scales showed that the four scales significantly correlated with each other (Table 2).

\section{RQ1}

To test whether the pace of life significantly differs across the country of origin (i.e., students with and without migration background), a multivariate analysis of variance was employed. The descriptive data given in Table 1 demonstrate significant differences across students without a migration background (from Germany), and students from other countries while controlling for adaption.

Adaption (represented by first-, second-, or third- year studies i.a. 1, 2 or 3 years length of stay at the current university) was included as a covariate, but revealed no significant difference, $p=.24, \eta^{2}<.04$. However, the migration was a significant variable when analyzing all variables together, $F_{\text {Wilks's }}$ ${ }_{\Lambda}(5,148)=3.29, p=.01, \eta^{2}=.10$. Investigating the individual variables, it turned out that only satisfaction with life was significantly different between the students from Germany $(M=24.22)$ and the students from other countries
$(M=21.71), p=.04, \eta^{2}=.03$. In other words, the pace of life, perceived stress, work-life balance, and health appeared invariant across students with different adaptation levels and a different country of origin.

\section{RQ2}

A series of correlational analyses were performed to answer RQ2. Full results of the correlational analyses are displayed in Table 2 .

The pace of life significantly correlated with work-life balance only, but not with life satisfaction, stress level, and health. This correlation of $r=.21$ was rather medium to small, according to Cohen (1992). On the individual-item level, the pace of life in terms of engagement with extracurricular activities correlated significantly with all other variables, $r=-.23-.30$. The pace of life in terms of sleep and being punctual significantly correlated with life satisfaction, stress, and health (see Table 2), but not with work-life balance. The pace of life in terms of how quickly students respond to email messages was significantly associated with stress, work-life balance, and health, but not with life satisfaction. The pace of life in terms of finishing a meal and becoming irritable when doing nothing is significantly interrelated only with one other variable each (for details of analyses, see Table 2). All significant correlations were between -.23 and -.19 or .17 and .30 , which can be interpreted as rather medium to small, according to Cohen (1992).

\section{RQ3}

Before the regression analysis was conducted, the length of stay at the current university and study major were converted into dummy variables to allow their inclusion in the regression model. Results can be found in Table 3, with Model 1 testing only the demographic variables age, gender, country of origin, adaption, and study major; Model 2 testing pace of life; and Model 3 introduced all other variables. With Model 1, only $10 \%$ of the variance was

Table 1

Descriptives of the Different Variables within Students from Germany or All Other Countries

\begin{tabular}{|c|c|c|c|}
\hline & Germany $(n=51) M(S D)$ & Other countries $(n=105) M(S D)$ & Total $(N=156) M(S D)$ \\
\hline Pace of life & $3.26(0.41)$ & $3.20(0.41)$ & $3.22(0.41)$ \\
\hline Perceived stress & $18.86(7.16)$ & $20.38(6.74)$ & $19.89(6.90)$ \\
\hline Work-life balance & $3.01(0.78)$ & $3.11(0.83)$ & $3.08(0.81)$ \\
\hline Health & $2.96(0.86)$ & $3.15(0.97)$ & $3.09(0.93)$ \\
\hline Satisfaction with life & $24.22(6.36)$ & $21.71(6.83)$ & $22.53(6.76)$ \\
\hline
\end{tabular}

Note. Groups differed significantly only with satisfaction with life, $p=.03$; all other $p \mathrm{~s} \geq .20$. 
Table 2

Pearson Correlations of the Pace of Life Scale and Single Items with Well-Being Variables

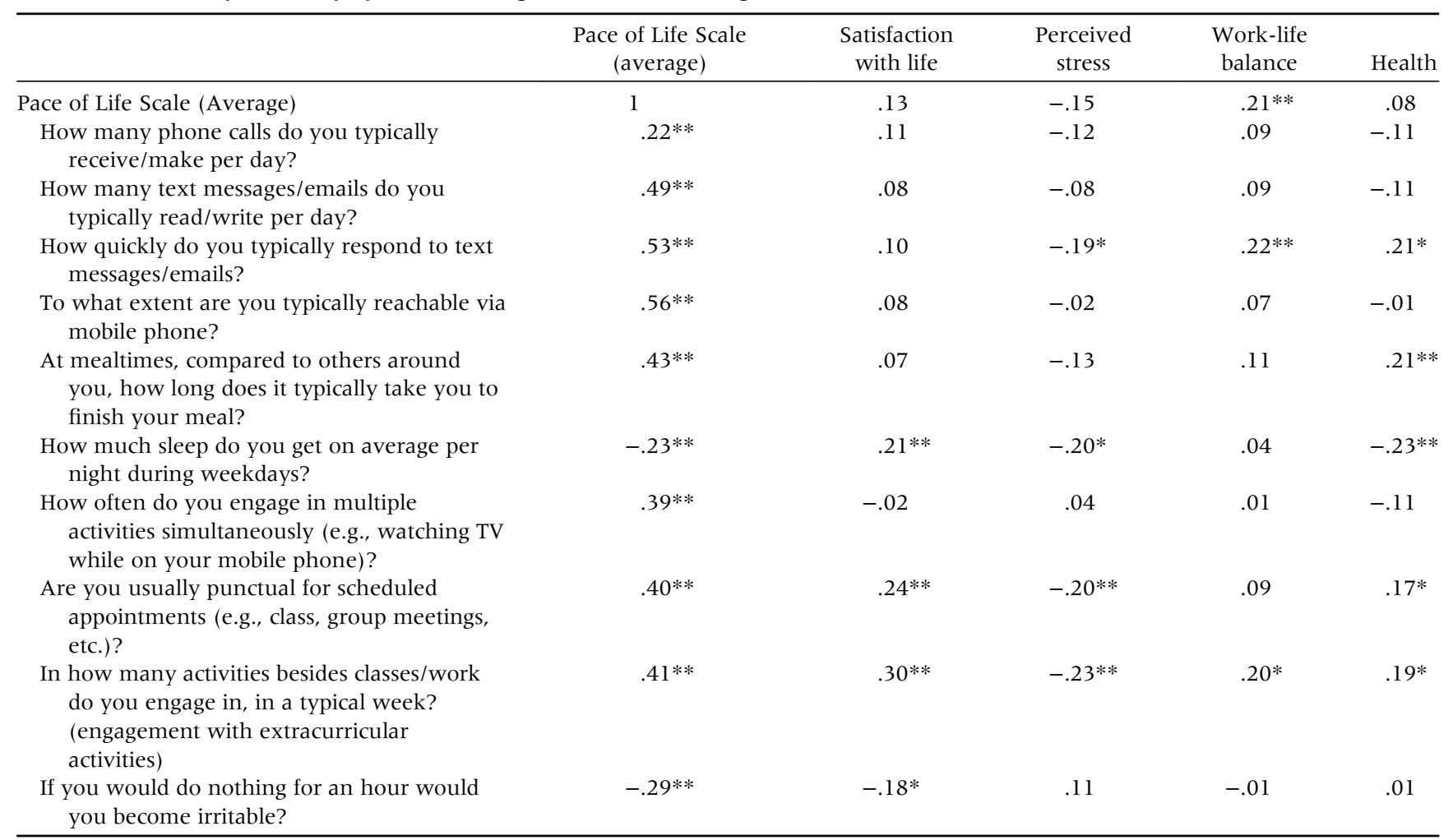

$* p<.05$.

$* * p<.01$.

explained, and only gender, but no other coefficients, was significant (validating earlier results from the analysis of variance/multivariate analysis of variance were also $10 \%$ of the variance could be explained by the sociodemographic variables.) In Model 2, all variables explained 13\% of the variance in stress, with the pace of life contributing

Table 3

Hierarchical Regression Analysis Predicting Perceived Stress (PSS)

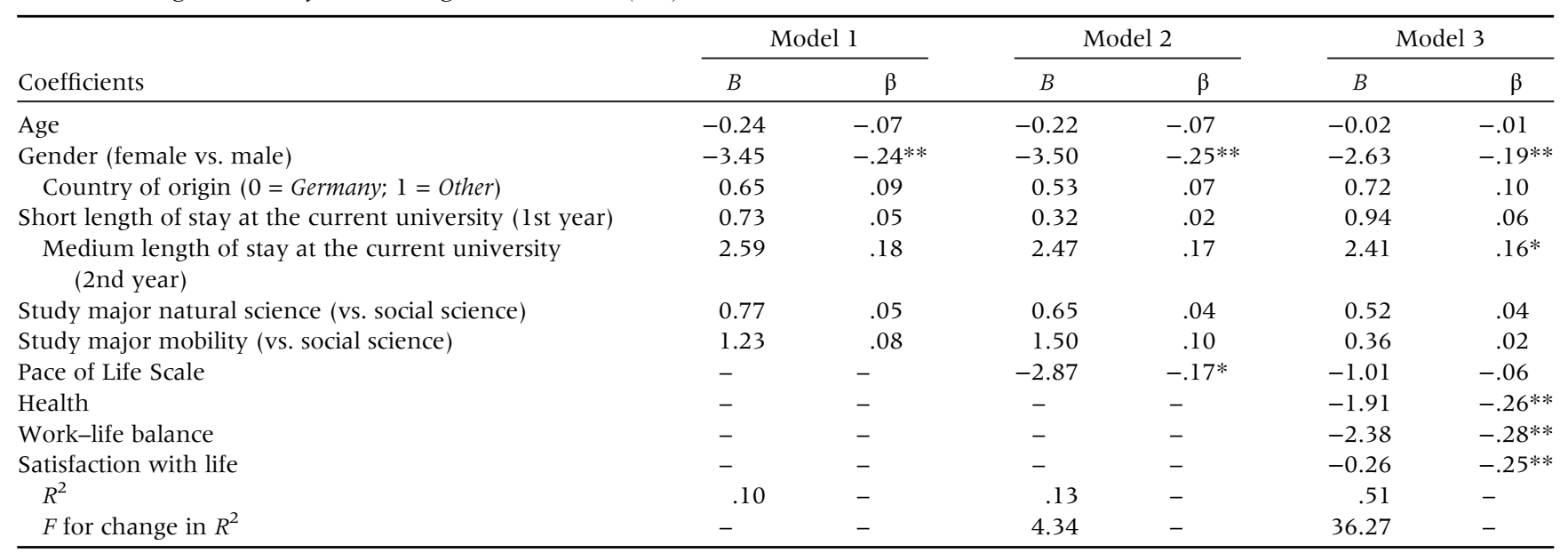

Note. Females reported more stress, the more adapted, the more stress, whereas pace of life was not significant in Model 3 (but was in Model 1 and Model 2), better health, more work-life balance, and more satisfaction with life was related to fewer stress.

$* p<.05$.

$* * p<.01$. 
significantly, $\beta=-.17, p=.04$. In Model 3, all additional variables (health, work-life balance, and life satisfaction) were significant, $p \mathrm{~s}<.01$, and overall, $51 \%$ of the variance of the reported stress could be explained by the investigated variables. However, the pace of life no longer contributed to any unique variance. This demonstrates that the pace of life, adaption, or the country of origin variables did not significantly explain variance in stress after controlling for age, gender, and study major as well as health, work-life balance, and life satisfaction. However, given that the pace of life correlated significantly with work-life balance (see Table 2) and that the pace of life correlated with perceived stress as long as work-life balance was not controlled for (see Models 2 and 3), one might speculate that the relationship between the pace of life and perceived stress is actually mediated by work-life balance: Students who experience a faster pace of life may cope with that lifestyle by more effective and strict time management, leading to greater work-life balance which in turn might reduce their perceived level of stress. To test this possibility, we ran a simple mediation analysis using PROCESS Model 4 (Hayes, 2018).

In the mediation analysis, we entered the pace of life as the independent variable, perceived stress as the dependent variable, and work-life balance as the potential mediator. There was limited evidence for a direct effect of the pace of life on perceived stress, $b=-2.656, S E=1.34, t$ $(155)=-1.97, p=.05,95 \%$ CI $[-5.313,0.001]$. However, according to many scholars, indirect mediation effects can still validly be interpreted, even in the absence of a strong direct effect between the independent and the dependent variables (Rucker, Preacher, Tormala, \& Petty, 2011; Zhao, Lynch, \& Chen, 2010).

After entering work-life balance as the mediator into the model, the pace of life significantly predicted work-life balance, $b=0.413, S E=0.16, t(155)=6.94, p<.001$, $95 \%$ CI $[0.102,0.723]$, which in turn predicted the perceived level of stress, $b=-4.58, S E=0.59, t$ $(155)=-7.81, p<.001,95 \%$ CI $[-5.739,-3,422]$. We found that the relationship between the pace of life and the perceived level of stress was indeed mediated by work-life balance with a significant indirect effect $b=-1.89$, $p<.001,95 \%$ CI $[-3.598,-0.463]$. In sum, this mediation effect provides evidence for our assumption that students cope with a faster pace of life by effective time management, leading to greater work-life balance and thus reducing the experienced level of stress.

\section{Discussion}

This study investigated the prevalence and correlates of the pace of life among university students with diverse cultural backgrounds via a cross-sectional questionnaire survey design. Results showed comparatively high stress levels among the investigated students $(M=15.60$, $S D=6.67$; Nordin \& Nordin, 2013, and $M=19.89$, $S D=6.90$; the current study). In contrast to previous studies (Levine \& Norenzayan, 1999), the pace of life appeared invariant across cultural backgrounds. The pace of life was significantly associated with work-life balance only, but not (directly) with life satisfaction, stress level, and health. The variables of health, work-life balance, and life satisfaction explained over half of the variance in stress after controlling for sociodemographic variables.

Regarding the examination of RQ1 in detail, one possible explanation why the pace of life did not vary greatly across cultural backgrounds might be that the students' fast adaptation could have negated all relationships between the pace of life and country of origin of the students. In other words, the perceived pace of life might depend more strongly on the society one lives in currently rather than on the previous cultural background one originally comes from. If all students adopt similar cultural orientations, no relationship between country of origin and pace of life can be expected. In addition, considering the findings of Taras, Steel, and Kirkman (2016), which indicated that within the country of origin differences are larger than betweencountry differences, it comes as no surprise that no relationship was found between country of origin and pace of life, as culture or acculturation was not measured on an individual level in this study.

In terms of a series of correlational investigations related to the pace of life, results from this study were not in line with prior findings that a faster pace of life is related to higher life satisfaction (i.e., quality of life) on the composite score level (Garhammer, 2002; Levine \& Norenzayan, 1999). However, satisfaction with life was significantly correlated with single indicators of the pace of life, such as punctuality and the number of activities involved in. The results furthermore suggested that more stress does not directly interrelate with a fast pace of life: Although the aggregate was not significantly interrelated, specific items of the pace of life revealed significance, such as the correlations between more sleep and less stress. This 
may be because those students who manage their tasks more effectively have more time to sleep and accordingly experience less stress.

Additionally, lower levels of stress were related to less time needed to respond to an email message, possibly for the simple reason that students who manage to respond to a message quickly have the time to relax afterward instead of regretting not having responded quicker and accordingly are less stressed. More punctual students feel less stressed, perhaps also because they feel that being on time helps enhance their sense of control, which reduces the stress level; whereas those who always feel being not punctual but "running late" perceive more stress due to this perception of being chased and maybe even overwhelmed. Additionally, those students feeling more stressed due to being not punctual may also perceive this as failed attempts to comply with time etiquette. This was corroborated by the detected mediation effect: Students seem to cope with a faster pace of life by effective time management, which also helps them to better balance work and life, and in turn reduce the experienced level of stress. However, it should be taken into account that this study only provided crosssectional data. However, other research (e.g., Adams \& Blair, 2019) also has revealed this in undergraduate engineering students' performance measured in grades: Individuals with more efficacious behavior felt less stressed and thereby had more time and the ability to better manage their tasks.

We did, however, find an indirect effect of the pace of life on the experienced level of stress, mediated by worklife-balance. This finding suggests that individuals with a faster pace of life tend to strictly schedule their lives, thus being able to maintain a better balance between work and private life (Levine, 1997). Likewise, students who engage in more leisure activities per week might be able to do so because they have a better work-life balance. In turn, the work-life balance might be better maintained because more leisure activities help to actually balance life and to effectively cope with stress. Yet, given the exploratory nature of our study, we regard this primarily as a hypothesis for future investigations. Such studies might directly assess time management and effective scheduling.

The findings regarding health are worthwhile to consider. Although the pace of life aggregate did not significantly correlate with the health status of students, it was found that the item regarding less sleep is indeed related to worse health. This comes as no surprise as sleep was found to impact health in previous literature (e.g., Shi et al., 2020). Furthermore, eating slower was related to better health. Additionally, Ohkuma et al. (2013) found the opposite relationship - that fast eating was related to cardiovascular risk factors. While their approach was based on objective health measures, the participants in this study were simply asked to rate their health. In addition, engaging in more leisure activities was related to better health. A simple reason for this relationship might be the higher chance that the participant engages in physical exercise, which was found to be positively related to health with ample evidence $(\mathrm{Ku}$, Hamer, Liao, Hsueh, \& Chen, 2020; Renninger et al., 2020; Thomas, Thirlaway, Bowes, \& Meyers, 2020). Students need to self-regulate to fit physical activity into a busy week schedule.

Regarding the research question on which of the variables explains the most variance in stress after testing the impact of the pace of life among students and controlling for age, gender, adaption, and study subject, the following can be concluded: Work-life balance explained most variance, with $\sim 8 \%$ of the variance, health explained $\sim 7 \%$, and satisfaction with life $\sim 6 \%$. The only other significant variables were gender, which explained about $4 \%$ of the variance, and a longer stay at the university with $\sim 3 \%$ explained variance. The pace of life was not significant if the main variables were included, indicating a high overlap in the variance of the pace of life with health, work-life balance, and satisfaction with life.

\section{Limitations and recommendations for further research}

The results of analyses including adaption should be treated with caution, as their measurements in this study were done using proxy variables asking about the length of stay at the current university. Results might change if adaption and home culture are measured by validated instruments. Another important limitation is that the pace of life items were self-developed and prevalidated with items gathered from relevant references. Further research can assess these variables more accurately by designing more systematic and well-validated tools to provide more conclusive findings. Furthermore, this study has certain limitations, suggesting cautious explanations for the results: As an exploratory study with a cross-sectional design with selfreported measures and multiple-correlations analyses, the results should not be taken as final explanations of causal relationships. Instead, all results from the present study can only reveal the correlational relationship among the pace of 
life related variables, and not only the significance level of the findings but also the practical relevance of the individual results should be checked, following Cohen (1992). Future studies should accordingly replicate the findings in longitudinal and interventional designs to reveal the underpinning causal mechanism related to the pace of life.

\section{Conclusion}

In summary, the present study revealed that the pace of life did not significantly vary with any demographic variables except gender. Age and the country of origin were not related to the pace of life. In addition, no differences in their pace of life were found between students with different lengths of stay at the current university and different study majors. Health or anti-stress programs of universities should thus not focus on particular groups but rather address all students. Relationships of the single pace of life items with the other variables and the mediation effect, however, justify the need to take pace of life into consideration, helping students to self-regulate even under stress to eat mindfully (with enough time), to sleep enough, and to perform leisure-time activities. As a result, students should better balance work and nonwork, reduce stress, promote their health, and be more satisfied with their life. Effective time and self-management can help with that.

\section{Disclosure of conflict of interest}

The authors declare that there are no conflicts of interest.

\section{Acknowledgments}

The authors would also like to thank Ronja Bellinghausen and Nara Skipper for proofreading this manuscript. Open access funding enabled and organized by Projekt DEAL.

\section{References}

Adams, R. V., \& Blair, E. (2019). Impact of time management behaviors on undergraduate engineering students' performance. SAGE Open, 9(1), 215824401882450. https://doi.org/10.1177/ 2158244018824506

American College Health Association (2018). American college health association-National College Health Assessment II: Reference group executive summary fall 2017 . Hanover, MD: American College Health Association.
Bornstein, M. H., \& Bornstein, H. G. (1976). The pace of life. Nature, 259, 557-559. https://doi.org/10.1038/259557a0

Burnett, A., DiTunnariello, N., \& DeGreeff, B. L. (2020). "I'm on a rollercoaster": Women's social construction of time. Communication Studies, 71, 148-166. https://doi.org/10.1080/ 10510974.2019.1702073

Cohen, J. (1992). A power primer. Psychological Bulletin, 112(1), 155-159. https://doi.org/10.1037/0033-2909.112.1.155

Cohen, S., \& Williamson, G. (1988). Perceived stress in a probability sample of the US. In S. Spacapam \& S. Oskamp (Eds.), The social psychology of health (pp. 31-67). Newbury Park, CA: Sage.

Cohen, S., Kamarck, T., \& Mermelstein, R. (1983). A global measure of perceived stress. Journal of Health and Social Behavior, 24, 385-396. https://doi.org/10.2307/2136404

Cotton, S. J., Dollard, M. F., \& de Jonge, J. (2002). Stress and student job design: Satisfaction, well-being, and performance in university students. International Journal of Stress Management, 9(3), 147-162. https://doi.org/10.1023/A:1015515714410

Garhammer, M. (2002). Pace of life and enjoyment of life. Journal of Happiness Studies, 3(3), 217-256. https://doi.org/10. 1023/A:1020676100938

Gleick, J. (1999). Faster: The acceleration of just about everything. New York, NY: Pantheon Books.

Goldbach, C., Hoffmann, C., Hoppe, J., Pitz, T., \& Thommes, K. (2020). The fast and the furious-An experimental investigation of the pace of life and risky speed choice in traffic. PLoS One, 15(7), e0236589. https://doi.org/10.1371/journal.pone.0236589

Güzel, H., \& Glazer, S. (2019). Demographic correlates of acculturation and sociocultural adaptation: Comparing international and domestic students. Journal of International Students, 9(4), 1074-1094. https://doi.org/10.32674/jis.v10i1.614

Hayes, A. F. (2018). Introduction to mediation, moderation, and conditional process analysis a regression-based approach. New York, NY: Guilford Press.

Hofstede, G. (2001). Culture's consequences: Comparing values, behaviors, institutions, and organizations across nations (2nd ed.). Thousand Oaks, CA: Sage.

Hofstede, G., Hofstede, G. J., \& Minkov, M. (2010). Cultures and organizations: Software of the mind (3rd ed.). New York, NY: McGraw-Hill.

Keinan, A., Bellezza, S., \& Paharia, N. (2019). The symbolic value of time. Current Opinion in Psychology, 26, 58-61. https://doi.org/10.1016/j.copsyc.2018.05.001

Ku, P. W., Hamer, M., Liao, Y., Hsueh, M. C., \& Chen, L. J. (2020). Device-measured light intensity physical activity and mortality: A meta-analysis. Scandinavian Journal of Medicine \& Science in Sports, 30, 13-24. https://doi.org/10.1111/sms.13557

Levine, R. V. (1997). A geography of time . New York, NY: Basic Books.

Levine, R. V., \& Bartlett, K. (1984). Pace of life, punctuality, and coronary heart disease in six countries. Journal of CrossCultural Psychology, 15, 233-255. https://doi.org/10.1177/ 0022002184015002009

Levine, R. V., \& Norenzayan, A. (1999). The pace of life in 31 countries. Journal of Cross-Cultural Psychology, 30, 178-205. https://doi.org/10.1177/0022022199030002003

Levine, R. V., Lynch, K., Miyake, K., \& Lucia, M. (1989). The type a city: Coronary heart disease and the pace of life. Journal 
of Behavioral Medicine, 12, 509-524. https://doi.org/10.1007/ bf00844822

Mathot, K. J., \& Frankenhuis, W. E. (2018). Models of pace-oflife syndromes (POLS): A systematic review. Behavioral Ecology and Sociobiology, 72(3), 41. https://doi.org/10.1007/ s00265-018-2459-9

Mei, Q., Li, C., Yin, Y., Wang, Q., Wang, Q., \& Deng, G. (2019). The relationship between the psychological stress of adolescents in school and the prevalence of chronic low back pain: A cross-sectional study in China. Child and Adolescent Psychiatry and Mental Health, 13(1), 24. https://doi.org/10. 1186/s13034-019-0283-2

Melnikov, V. R., Krzhizhanovskaya, V. V., Lees, M. H., \& Sloot, P. M. (2020). The impact of pace of life on pedestrian heat stress: A computational modelling approach. Environmental Research, 186, 109397. https://doi.org/10.1016/j.envres.2020.109397

Nagy, P., Eschrich, J., \& Finn, E. (2020). Time hacking: How technologies mediate time. Information, Communication \& Society, 1-15. https://doi.org/10.1080/1369118X.2020.1758743

Nordin, M., \& Nordin, S. (2013). Psychometric evaluation and normative data of the Swedish version of the 10-item perceived stress scale. Scandinavian Journal of Psychology, 54(6), 502-507. https://doi.org/10.1111/sjop.12071

Ohkuma, T., Fujii, H., Iwase, M., Kikuchi, Y., Ogata, S., Idewaki, Y., ... Kitazono, T. (2013). Impact of eating rate on obesity and cardiovascular risk factors according to glucose tolerance status: The Fukuoka diabetes registry and the Hisayama study. Diabetologia, 56, 70-77. https://doi.org/10.1007/s00125012-2746-3

Pascoe, M. C., Hetrick, S. E., \& Parker, A. G. (2020). The impact of stress on students in secondary school and higher education. International Journal of Adolescence and Youth, 25, 104-112. https://doi.org/10.1080/02673843.2019.1596823

Pavot, W., \& Diener, E. (2008). The Satisfaction With Life Scale and the emerging construct of life satisfaction. Journal of Positive Psychology, 3, 137-152. https://doi.org/10.1080/1743 9760701756946

Redfield, R., Linton, R., \& Herskovits, M. J. (1936). Memorandum for the study of acculturation. American Anthropologist, 38, 149-152. https://doi.org/10.1525/aa.1936.38.1.02a00330

Renninger, M., Hansen, B. H., Steene-Johannessen, J., Kriemler, S., Froberg, K., Northstone, K., ... International Children's Accelerometry Database (ICAD) Collaborators (2020). Associations between accelerometry measured physical activity and sedentary time and the metabolic syndrome: A meta-analysis of more than 6000 children and adolescents. Pediatric Obesity, 15(1), e12578. https://doi.org/10.1111/ijpo.12578

Roberti, J. W., Harrington, L. N., \& Storch, E. A. (2006). Further psychometric support for the 10-item version of the perceived stress scale. Journal of College Counseling, 9, 135-147. https://doi.org/10.1002/j.2161-1882.2006.tb00100.x

Rosa, H. (2003). Social acceleration: Ethical and political consequences of a desynchronized high-speed society. Constellations, 10, 3-33. https://doi.org/10.1111/1467-8675.00309

Rosenman, R. H. (1990). Type a behaviour pattern: A personal overview. Journal of Social Behavior and Personality, 5(1), 1-24. $\mathrm{https}$ //search.proquest.com/docview/1292329776?accountid=14136

Royauté, R., Berdal, M. A., Garrison, C. R., \& Dochtermann, N. A. (2018). Paceless life? A meta-analysis of the pace-of-life syndrome hypothesis. Behavioral Ecology and Sociobiology, 72(3), 64. https://doi.org/10.1007/s00265-0182472-z

Rucker, D. D., Preacher, K. J., Tormala, Z. L., \& Petty, R. E. (2011). Mediation analysis in social psychology: Current practices and new recommendations. Social and Personality Psychology Compass, 5, 359-371. https://doi.org/10.1111/j.17519004.2011.00355.x

Shi, T., Min, M., Sun, C., Zhang, Y., Liang, M., \& Sun, Y. (2020). Does insomnia predict a high risk of cancer? A systematic review and meta-analysis of cohort studies. Journal of Sleep Research, 29(1), e12876. https://doi.org/10.1111/jsr.12876

Sullivan, O., \& Gershuny, J. (2001). Cross-national changes in time-use: Some sociological (hi) stories re-examined. The British Journal of Sociology, 52, 331-347. https://doi.org/10.1080/ 00071310120045015

Syrek, C., Bauer-Emmel, C., Antoni, C., \& Klusemann, J. (2011). Entwicklung und Validierung der Trierer Kurzskala zur Messung von Work-Life Balance (TKS-WLB). Diagnostica, 57, 134-145. https://doi.org/10.1026/0012-1924/a000044

Taras, V., Steel, P., \& Kirkman, B. L. (2016). Does country equate with culture? Beyond geography in the search for cultural boundaries. Management International Review, 56, 455-487. https://doi.org/10.1007/s11575-016-0283-x

Thomas, J., Thirlaway, K., Bowes, N., \& Meyers, R. (2020). Effects of combining physical activity with psychotherapy on mental health and well-being: A systematic review. Journal of Affective Disorders, 265, 475-485. https://doi.org/10.1016/j. jad.2020.01.070

Thomeé, S., Härenstam, A., \& Hagberg, M. (2011). Mobile phone use and stress, sleep disturbances, and symptoms of depression among young adults - a prospective cohort study. BMC Public Health, 11(66), 1-11. https://doi.org/10.1186/1471-2458-11-66

van de Vliert, E., \& Janssen, O. (2002). "Better than" performance motives as roots of satisfaction across more and less developed countries. Journal of Cross-Cultural Psychology, 33 (4), 380-397. https://doi.org/10.1177/00222102033004002

Wang, C.-C. D., \& Mallinckrodt, B. (2006). Acculturation, attachment, and psychosocial adjustment of Chinese/Taiwanese international students. Journal of Counseling Psychology, 53(4), 422-433. https://doi.org/10.1037/0022-0167.53.4.422

Ward, C., \& Kennedy, A. (1999). The measurement of sociocultural adaptation. International Journal of Intercultural Relations, 23, 659-677. https://doi.org/10.1016/S0147-1767(99)00014-0

Ware, J. E., \& Sherbourne, C. D. (1992). The MOS 36-item short-form health survey (SF-36): I. conceptual framework and item selection. Medical Care, 30, 473-483. https://doi.org/10. 1007/BF03260127

Werner, C. M., Altman, I., \& Oxley, D. (1985). Temporal aspects of homes: A transactional perspective. In I. Altman \& C. M. Werner (Eds.), Home environments: Vol. 8. Human behavior and environment: Advances in theory and research (pp. 1-32). New York, NY: Plenum. https://doi.org/10.1007/978-1-4899-2266-3_1

Whoqol Group (1998). Development of the World Health Organization WHOQOL-BREF quality of life assessment. Psychological Medicine, 28, 551-558. https://doi.org/10.1017/ s0033291798006667

Wilson, J., Ward, C., Fetvadjiev, V. H., \& Bethel, A. (2017). Measuring cultural competencies: The development and 
validation of a revised measure of sociocultural adaptation. Journal of Cross-Cultural Psychology, 48(10), 1475-1506. https://doi.org/10.1177/0022022117732721

Wilton, L., \& Constantine, M. G. (2003). Length of residence, cultural adjustment difficulties, and psychological distress symptoms in Asian and Latin American international college students. Journal of College Counseling, 6(2), 177-186. https://doi.org/10.1002/j.2161-1882.2003.tb00238.x
Xing, X., Popp, M., \& Price, H. (2020). Acculturation strategies of Chinese University students in the United States. Journal of Advances in Education Research, 5(1), 11-24. https://doi.org/ 10.22606/jaer.2020.51002

Zhao, X., Lynch, J. G., Jr., \& Chen, Q. (2010). Reconsidering baron and Kenny: Myths and truths about mediation analysis. Journal of Consumer Research, 37, 197-206. https://doi.org/ $10.1086 / 651257$ 